\title{
Bienvenue à Marseille pour le Congrès 2017 de la Société internationale francophone d'éducation médicale (SIFEM)
}

Depuis 2012, le Centre d'innovation pédagogique et d'évaluation (CIPE) d'Aix-Marseille Université a suscité et accompagne les initiatives des enseignants chercheurs dans les disciplines de santé, particulièrement impliqués. C'est pourquoi l'Université d'Aix-Marseille est particulièrement heureuse de vous accueillir à l'occasion de ce congrès, sur son campus de la Timone qui regroupe les facultés de médecine, pharmacie et odontologie.

Les congrès sont des opportunités rares de partages. Il s'agit d'abord du partage d'expertise, grâce aux interventions et aux échanges avec des conférenciers internationalement reconnus pour leurs activités de recherche et de formation. Il s'agit ensuite du partage de savoirs, à l'occasion d'ateliers de formation organisés sur le plan pédagogique selon des principes qui permettent la coconstruction de connaissances entre les participants et avec les animateurs. Il s'agit enfin du partage d'expériences, grâce à la présentation, sous la forme de communications orales et affichées, de résultats originaux de recherche et de dispositifs innovants de formation, que plusieurs d'entre vous ont eu l'opportunité de concevoir et de mettre en œuvre au sein de leurs institutions.

De nombreux intervenants, issus de toute la francophonie, ont accepté de s'inscrire dans ces diverses perspectives de partages. Nous avons notamment observé cette année un record en matière de soumissions de communications et d'ateliers. Nous vous encourageons d'ailleurs à récompenser les meilleures propositions, en déposant à la borne d'accueil du congrès les bulletins de vote que vous trouverez à la fin de ce fascicule.

Ces indicateurs témoignent du dynamisme du champ de l'éducation des sciences de la santé, qui connaît un essor important depuis le début des années 2000. Ce développement a été accentué par les réformes curriculaires et pédagogiques majeures qui ont marqué de nombreux dispositifs de formation des professionnels de la santé au cours des dernières années.

Tous les ingrédients sont ainsi réunis pour que vous preniez plaisir à évoluer pendant ces trois journées dans un environnement que nous vous souhaitons riche en rencontres et en opportunités d'apprentissages!

Ce congrès est aussi celui du redéploiement de la Société internationale francophone d'éducation médicale (SIFEM).

Elle a pour mission de promouvoir la santé par la formation des professionnels et la recherche en éducation. En tant que société savante elle vise à regrouper, au sein du monde francophone, les personnes impliquées dans la formation et la recherche au sein des formations professionnelles en santé.

Elle vit actuellement un redéploiement qui s'articule autour de quelques axes:

- rejoindre l'ensemble des professionnels de santé intéressés par la pédagogie;

- contribuer à créer un congrès international francophone annuel en pédagogie des formations professionnelles en santé;

- diffuser largement Pédagogie Médicale, revue scientifique et organe officiel d'expression de la SIFEM;

- répondre aux besoins de nos membres, notamment par les activités de groupes thématiques (raisonnement clinique, mesure et évaluation, communication professionnelle en santé, éthique des curriculums, responsabilité sociale).

La SIFEM partage les valeurs suivantes: innovation, collaboration interprofessionnelle, rigueur, éthique et équité.

L'équipe de direction de la SIFEM a hâte de vous rencontrer à Marseille.

$$
\begin{array}{r}
\text { Jacques DÉJOU } \\
\text { Président d'honneur du congrès } \\
\text { Bernard CHARLIN } \\
\text { Président de la SIFEM } \\
\text { Anne DEMEESTER } \\
\text { Thierry PELACCIA }
\end{array}
$$$$
\text { Présidente du comité d'organisation du congrès }
$$$$
\text { Président du comité scientifique du congrès }
$$ 